See Article page e105.

\section{Commentary: The jury is still out}

\author{
Dawn S. Hui, MD, ${ }^{\mathrm{a}}$ and Richard Lee, MD, MBA ${ }^{\mathrm{b}}$
}

In 2016, Drs Reardon and Thourani noted that for the first time, the national volume of transcatheter aortic valve replacement (TAVR) exceeded that of surgical aortic valve replacement (SAVR), at 37,113 versus 28,037. ${ }^{1}$ In March of this year, Dr Michael Mack forecasted that TAVR volumes would approach 100,000 in 2020, driven by approval for low-risk patients. ${ }^{2}$ Also in 2020, results for low-risk patients in the Placement of Aortic Transcatheter Valves (PARTNER) 3 trial were updated to report 2-year outcomes. Notably, valve thrombosis, by the VARC-2 (Valve Academic Research Consortium) definition, more than doubled in TAVR patients to $2.6 \%$ at 2 years. ${ }^{3}$ In studies that use computed tomography (CT) screening for valve thrombosis, the prevalence has been reported to be much higher, at $9.3 \%$ to $13 \%{ }^{4-6}$ Valve thrombosis is associated with higher rates of stroke and transient ischemic attack..$^{5-7}$

The near-tripling of annual TAVR volumes combined with these early signals of valve thrombosis render an urgent need to investigate the underlying mechanisms. In this issue of the Journal, Trusty and colleagues ${ }^{8}$ report on the relationship between flow stasis and valve thrombosis. There is much that is well done in this study: patients were recruited from an observational registry reflecting real-world practices, patient-specific native and deployment anatomy were modeled, and 4-dimensional imaging was an advancement over previous in the field. However, there are some fundamentals that leave much to be desired. First, the sample size of 23 is a jarring contrast not only to the volumes mentioned above, but also to the registry ${ }^{6}$ from which they were drawn, representing only $3.7 \%$ of the interpretable scans. The RESOLVE registry as a source is itself somewhat problematic: CT timing was not prespecified,

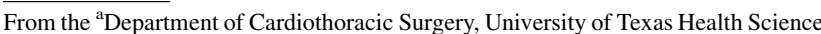
Center at San Antonio, San Antonio, Tex and bivision of Cardiothoracic Surgery, Department of Surgery, Medical College of Georgia, Augusta University, Augusta, Ga. Disclosures: The author reported no conflicts of interest.

The Journal policy requires editors and reviewers to disclose conflicts of interest and to decline handling or reviewing manuscripts for which they may have a conflict of interest. The editors and reviewers of this article have no conflicts of interest.

Received for publication Nov 16, 2020; revisions received Nov 16, 2020; accepted for publication Nov 17, 2020; available ahead of print Nov 28, 2020.

Address for reprints: Richard Lee, MD, MBA, Department of Surgery, Medical College of Georgia, 1120 15th St, BA-4300, August, GA 30912; (E-mail: richardleemdmba@gmail.com or RICKLEE@augusta.edu).

J Thorac Cardiovasc Surg 2022;164:e119-20 $0022-5223 / \$ 36.00$

Copyright $(2020$ Published by Elsevier Inc. on behalf of The American Association for Thoracic Surgery

https://doi.org/10.1016/j.jtcvs.2020.11.082
}

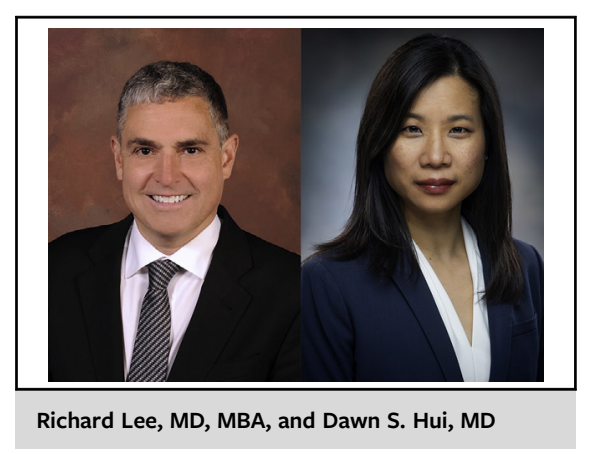

\author{
CENTRAL MESSAGE \\ Sophisticated modeling and flow \\ study techniques represent ad- \\ vances in the study of prosthetic \\ valve thrombosis, but basic prin- \\ ciples are still needed to reveal \\ mechanistic insights.
}

enrollment was nonconsecutive, patients were enrolled at various time points relative to TAVR, renal impairment was (understandably) an exclusion criteria, and follow-up imaging was "clinically driven" rather than driven by protocol. Whereas Trusty's abstract asserts that "patients were selected to minimize potential confounding factors related to valve thrombosis," this is not expounded upon in the manuscript and is a curious statement, given that so little is known about valve thrombosis itself, to say nothing of potential confounders. A great deal of anatomic measurements are provided, but the only individual patient-level clinical variable provided is cardiac output. Given that previous studies have found reduced low-flow low-gradient aortic stenosis in balloon-expandable valves ${ }^{5}$ and reduced ejection fraction in all TAVR valves ${ }^{7}$ to independently predict valve thrombosis, it would have been beneficial to include these clinical parameters.

The generalizability of the data for these 23 patients is thus questionable, especially given that mean follow-up was only 1 month. This is important, as the recent CT substudy of the PARTNER 3 trial showed that the incidence of hypoattenuated leaflet thickening (HALT), a marker of valve thrombosis, increased from $10 \%$ at 30 days to $24 \%$ at 1 year. $^{9}$ More than one-half of patients with 30-day HALT had spontaneous resolution, but new HALT appeared in $21 \%$ at 1 year. In contrast, Trusty and colleagues found that all patients had some degree of thrombosis and that the prevalence of HALT was noticeably higher at $74 \%$. Future studies should account for the dynamic nature of 
valve thrombosis and HALT, which will require a deeper understanding of this relationship over time.

Furthermore, although Trusty and colleagues used detailed patient-specific modeling, the use of Parafilm to model aortic valve leaflets lacks verisimilitude for degenerative aortic stenosis. We found the authors' rationale that "leaflet thickness should not substantially affect neosinus flow status" theoretical and unsupported by any clear data at this time. At this nascent stage of understanding postTAVR hemodynamics, we urge more studies before incorporating such assumptions into study designs. Finally, the lack of a control group with SAVR patients is a limitation. The findings of this study can only be applied to the neosinus created by the TAVR stent frame. We have known for nearly a decade that early valve thrombosis can occur with surgical bioprostheses, ${ }^{10}$ and more recent attention to the issue has also demonstrated CT-detected valve thrombosis in surgical valves, with an equivalent rate to TAVR valves at 1 year (PARTNER 3 substudy). ${ }^{9}$

Trusty and colleagues' perspective statement for readers is that flow stasis is significantly correlated with thrombus volume. In a sense, this finding may seem intuitive as it comes from one of the pillars of Virchow's triad. However, readers searching for mechanistic insight into how thrombus formation begins should be aware that this study does not address the question. Other factors that may contribute to thrombus formation include additional flow dynamic parameters such as shear stress, device and deployment-related factors, and patient characteristics. The univariate analysis and small sample size of this study cannot model the potentially complex arrangement of factors leading to thrombus formation. It does not explain how some patients may have spontaneous resolution while others develop late thrombosis. Just as the jury remains out on low-risk TAVR durability, so does it remain out for the underlying mechanism of valve thrombosis, the two being intertwined.

\section{References}

1. Reardon MJ, Thourani VH. Commentary: racing to the rubicon. J Thorac Cardiovasc Surg. 2018;155:1457-8.

2. Jancin B. Who'll get SAVR in 2020? Cardiology news. February 3, 2020. Available at: https://www.mdedge.com/cardiology/article/216594/interventionalcardiology-surgery/wholl-get-savr-2020. Accessed November 14, 2020.

3. Kalvaitus K. New insights on low-risk TAVR vs surgery: PARTNER 3 at 2 years. CardiologyToday. March 29, 2020. Available at: https://www.healio.com/news/ cardiac-vascular-intervention/20200329/new-insights-on-lowrisk-tavr-vs-surgerypartner-3-at-2-years. Accessed November 14, 2020.

4. Yanagisawa R, Tanaka M, Yashima F, Arai T, Jinzaki M, Shimizu H, et al. Early and late leaflet thrombosis after transcatheter aortic valve replacement. Circ Cardiovasc Interv. 2019;12:e07349.

5. Chakravarty T, Søndergaard L, Friedman J, De Backer O, Berman D, Kofoed KF, et al. Subclinical leaflet thrombosis in surgical and transcatheter bioprosthetic aortic valves: an observational study. Lancet. 2017;389:2383-92.

6. Woldendorp K, Doyle MP, Black D, Ng M, Keech A, Grieve SM, et al. Subclinical valve thrombosis in transcatheter aortic valve implantation: a systematic review and meta-analysis. J Thorac Cardiovasc Surg. February 19, 2020 [Epub ahead of print].

7. Rashid HN, Gooley RP, Nerlekar N, Ihdayhid AR, McCormick LM, Nasis A, et al. Bioprosthetic aortic valve leaflet thrombosis detected by multidetector computed tomography is associated with adverse cerebrovascular events: a meta-analysis of observational studies. Eurointervention. 2018;13:e1748-55.

8. Trusty PM, Bhat SS, Sadri V, Salim MT, Funnell E, Kamioka N, et al. The role of flow stasis in transcatheter aortic valve leaflet thrombosis. J Thorac Cardiovasc Surg. 2022;164:e105-17.

9. Makkar RR, Blanke P, Leipsic J, Thourani V, Chakravarty T, Brown D. Subclinical leaflet thrombosis in transcatheter and surgical bioprosthetic valves: PARTNER 3 cardiac computed tomography substudy. J Am Coll Cardiol. 2020;75: 3003-15.

10. Brown ML, Park SJ, Sundt TM, Schaff HV. Early thrombosis risk in patients with biologic valves in the aortic position. J Thorac Cardiovasc Surg. 2012;144: 108-11. 